\title{
Leptonic, semileptonic and rare charm decays at the BES-III experiment
}

\section{Gang RONG*}

(On behalf of the BESIII Collaboration $)^{\dagger}$

Institute of High Energy Physics, CAS, Beijing, China

E-mail: rongg@ihep.ac.cn

Using data samples taken with the BES-III detector at the center-of-mass energies of $\sqrt{s}=3.773$ $\mathrm{GeV}, \sqrt{s}=4.009 \mathrm{GeV}$ and $\sqrt{s}=4.599 \mathrm{GeV}$, we studied leptonic, semileptonic and rare charm decays. From leptonic $D^{+} \rightarrow \mu^{+} v_{\mu}$ decays, we determined the most precise $D^{+}$-meson decay constant $f_{D^{+}}=\left(203.2 \pm 5.3_{\text {stat }} \pm 1.8_{\text {syst }}\right) \mathrm{MeV}$ and the most precise Cabibbo-Kobayashi-Maskawa (CKM) quark mixing matrix element $\left|V_{\text {cd }}\right|=0.2210 \pm 0.0058_{\text {stat }} \pm 0.0047_{\text {syst }}$. From the semileptonic $D^{0} \rightarrow K^{-} e^{+} v$ and $D^{0} \rightarrow \pi^{-} e^{+} v$ decays, we determined the most precise $D$-meson decay form factors $f_{+}^{K}(0)=0.7368 \pm 0.0026_{\text {stat }} \pm 0.0036_{\text {syst }}$ and $f_{+}^{\pi}(0)=0.6372 \pm 0.0080_{\text {stat }} \pm$ $0.0044_{\text {syst }}$, as well as the CKM quark mixing matrix elements $\left|V_{\mathrm{cs}}\right|=0.9601 \pm 0.0058_{\exp } \pm$ $0.0230_{\mathrm{LQCD}}$ and $\left|V_{\mathrm{cd}}\right|=0.2155 \pm 0.0030_{\mathrm{exp}} \pm 0.0094_{\mathrm{LQCD}}$. These decay constant and form factors could be used to incisively validate Lattice QCD calculations of these quantities, in turn, to improve measurements of $C P$-violation in $B$ decays. In addition, we measured the form factors for semileptonic $D$ decays containing a vector meson in the final states, and searched for the rare $D$ decays containing $e^{+} e^{-}$or $e^{+} e^{+}$in the final state from the data taken at $3.773 \mathrm{GeV}$. Using the data taken at $\sqrt{s}=4.599 \mathrm{GeV}$ we made a first measurement of semileptonic $\Lambda_{c}^{+} \rightarrow \Lambda e^{+} v_{e}$ decays. In this article we briefly report these results.

VIII International Workshop On Charm Physics

5-9 September, 2016

Bologna, Italy

*Speaker.

${ }^{\dagger}$ The work is supported in part by the National Natural Science Foundation of China (NSFC) under Contract No. 10935007, National Key Basic Research Program of China under Contracts No. 2009CB825204 and No. 2015CB856700. This work is also supported in part by the CAS Center for Excellence in Particle Physics (CCEPP). 


\section{Introduction}

The leptonic and semileptonic charm decays play a crucial role in understanding of both the strong and week interactions. These decays provide important supporting measurements for studies of $C P$-violation in $B$ meson decays. These measurements are needed to validate theoretical calculations ${ }^{1}$ of the decay constants and form factors of the $D$ and $B$ mesons. On the other hand these decays supply a very good place for measurements of the Cabibbo-Kobayashi-Maskawa (CKM) quark mixing matrix elements $\left|V_{\mathrm{cd}}\right|$ and $\left|V_{\mathrm{cs}}\right|$, with which one can check the unitarity of the CKM matrix and search for new physics (NP) beyond the Standard Model (SM) of particle physics.

In recent years, LQCD has provided calculations of charm-sector decay constants and form factors with steadily increasing precision. With these improvements in precision, experimental validation of the computed results are more and more important. At present, the main uncertainty of the apex of the $B_{d}$ unitarity triangle (UT) of $B$ meson decays is dominated by the theoretical errors in the LQCD determinations of the $B$ meson decay constants $f_{B_{(s)}}$ and decay form factors $f_{+}^{B \rightarrow \pi}(0)$ [1]. Precision measurements of the charm-sector decay constants $f_{D_{(s)}^{+}}$and form factors $f_{+}^{K(\pi)}\left(q^{2}\right)$ can be used to establish the level of reliability of LQCD calculations of $f_{B_{(s)}}$ and $f_{+}^{B \rightarrow \pi}(0)$. If the LQCD calculations of $f_{D_{(s)}^{+}}$and $f_{+}^{K(\pi)}\left(q^{2}\right)$ agree well with measured $f_{D_{(s)}^{+}}$and $f_{+}^{K(\pi)}\left(q^{2}\right)$ values, the LQCD calculations of the decay constants for $B_{(s)}$ meson leptonic decays, and the form factors for $B$ meson semileptonic decays can be more confidently used to improve measurements of $B_{(s)} \bar{B}_{(s)}$ mixing rates and $B$ meson semileptonic decay rates. The improved measurements of the $B_{(s)} \bar{B}_{(s)}$ mixing and $B$ meson semileptonic decay rates would, in turn, improve the determination of the $B_{d} \mathrm{UT}$, from which one can more precisely test the SM description of quark mixing and $C P$-violation, and thereby maximizing the sensitivity of quark flavor physics to NP. So precision measurements of $f_{D_{(s)}^{+}}$and $f_{+}^{K(\pi)}\left(q^{2}\right)$ are very important for precision test of the SM and searching for NP.

In the last six years we precisely measured these charm-sector decay constant $f_{D^{+}}$and form factors $f_{+}^{K(\pi)}(0)$, as well as the CKM matrix elements $\left|V_{\mathrm{cd}}\right|$ and $\left|V_{\mathrm{cs}}\right|$. In this article we briefly report these measurements.

\section{The experiment, data samples and analysis technique}

The BES-III is a general magnetic detector working at a double-ring $e^{+} e^{-}$collider operating in center-of-mass energy between $2 \sim 4.6 \mathrm{GeV}$, located at the Institute of High Energy Physics (IHEP), Beijing. The peak luminosity of the machine at $3.773 \mathrm{GeV}$ is $1 \times 10^{33} \mathrm{~cm}^{-2} \mathrm{~s}^{-1}$. With this detector we accumulated $2.92 \mathrm{fb}^{-1}, 0.48 \mathrm{fb}^{-1}$, and $0.57 \mathrm{fb}^{-1}$ of data at center-of-mass energies $\sqrt{s}=3.773$ $\mathrm{GeV}, \sqrt{s}=4.009 \mathrm{GeV}$, and $\sqrt{s}=4.599 \mathrm{GeV}$, respectively. At these energies the $D \bar{D}$ mesons, $D_{s}^{+} D_{s}^{-}$mesons and $\Lambda_{c}^{+} \bar{\Lambda}_{c}^{-}$baryons are produced in $e^{+} e^{-}$annihilation. This allows us use a double tag analysis technique to study leptonic and semileptonic charm decays.

In data analysis, taking $D^{+}$meson as an example, we first selected the $D^{-}$meson sample from its hadronic decay modes, which is called single $D^{-}$tag or singly tagged $D^{-}$meson, and

\footnotetext{
${ }^{1}$ The QCD technologies such as Lattice QCD (LQCD), QCD sumrules and some other theoretical approaches can calculate the decay constants and form factors of the $D$ and $B$ mesons.
} 
then selected the leptonic or semileptonic $D^{+}$decays in the system recoiling against the single $D^{-}$tags. To select the leptonic and semileptonic $D^{+}$decays with missing neutrino, we calculated $\mathrm{U}_{\text {miss }}=\mathrm{E}_{\text {miss }}-\mathrm{p}_{\text {miss }}$ or $\mathrm{M}^{2}{ }_{\text {miss }}=\mathrm{E}_{\text {miss }}^{2}-\mathrm{p}_{\text {miss }}^{2}$, where $\mathrm{E}_{\text {miss }}$ and $\mathrm{p}_{\text {miss }}$ are missing energy and missing momentum of the event in question. For the leptonic and semileptonic $D^{+}$decay events, the $\mathrm{U}_{\text {miss }}$ or $\mathrm{M}_{\text {miss }}^{2}$ should be zero. So, from distributions of $\mathrm{U}_{\text {miss }}$ or $\mathrm{M}_{\text {miss }}^{2}$ for the singly tagged $D^{-}$events we can get the number of signal events for the leptonic or semileptonic $D^{+}$decays, and then measure its decay rate. This double analysis technique is extended to measurements of $D^{0}, D_{s}^{+}$and $\Lambda_{c}^{+}$ decays.

\section{Leptonic decays}

In the SM of particle physics, the charged $D_{(s)}^{+}$meson can decay into a charged lepton and its associated neutron when the $c$ quark and the $\bar{d}$ quark or $\bar{s}$ quark annihilate via a virtual $W^{+}$ boson. To the lowest order, the decay width is proportional to the square of the product of the decay constant $f_{D_{(s)}^{+}}$times $\left|V_{c d(s)}\right|$, and is given by

$$
\Gamma\left(D_{(s)}^{+} \rightarrow \ell v\right)=\frac{G_{F}^{2}}{8 \pi} m_{\ell}^{2} M_{D_{(s)}^{+}}\left(1-\frac{m_{\ell}^{2}}{M_{D_{(s)}^{+}}^{2}}\right)^{2} f_{D_{(s)}^{+}}^{2}\left|V_{c d(s)}\right|^{2},
$$

where $G_{F}$ is the Fermi coupling constant, $M_{D_{(s)}^{+}}$is the $D_{(s)}^{+}$mass, $m_{\ell}$ is the mass of the charged lepton in the final state. Hence the leptonic $D_{(s)}^{+}$decay provides us an opportunity to measure the decay constants and the magnitudes of CKM element $V_{c d(s)}$.

\subsection{Leptonic $D^{+}$decays}

The first result of leptonic charm decays from the BES-III experiment is about study of $D^{+} \rightarrow \mu^{+} v_{\mu}$. At Charm 2012 held in Hawaii we reported measurements of branching fraction for $D^{+} \rightarrow \mu^{+} v_{\mu}$ decay and decay constant $f_{D^{+}}$, and we also reported determination of $\left|V_{c d}\right|$ which was determined with the leptonic $D^{+}$decay for the first time in the world [2]. To measure these quantities we used the double tag technique to reconstruct the $D^{+} \rightarrow \mu^{+} v_{\mu}$ decays from the data accumulated at $3.773 \mathrm{GeV}$. As study of $D^{+} \rightarrow \mu^{+} v_{\mu}$ decay performed at BES-II experiment [3], nine hadronic $D^{-}$decay modes $K^{+} \pi^{-} \pi^{-}, K_{S}^{0} \pi^{-}, K_{S}^{0} K^{-}, K^{+} K^{-} \pi^{-}, K^{+} \pi^{-} \pi^{-} \pi^{0}, \pi^{+} \pi^{-} \pi^{-}, K_{S}^{0} \pi^{-} \pi^{0}$, $K^{+} \pi^{-} \pi^{-} \pi^{-} \pi^{+}$and $K_{S}^{0} \pi^{-} \pi^{-} \pi^{+}$were used to accumulate the single $D^{-}$tags in this analysis. The signal of $D^{-}$tags are clearly observed in the beam energy constrained mass $\mathrm{M}_{\mathrm{BC}}$ spectra as shown in Fig. 1. A maximum likelihood fit to these $\mathrm{M}_{\mathrm{BC}}$ spectra yields $(170.31 \pm 0.34) \times 10^{4}$ single $D^{-}$ tags from this data. The $D^{+} \rightarrow \mu^{+} v_{\mu}$ decays were selected in the recoil side of the single $D^{-}$tags by requiring no extra tracks except a muon identified by muon chamber. The information of the neutrino was inferred from the variable of missing mass squared $\mathrm{M}_{\text {miss }}^{2}$ as defined in Section 2. Figure 2 shows the distribution of $\mathrm{M}_{\text {miss }}^{2}$, where signal is clearly peaked around 0 . The peak around $0.25 \mathrm{GeV}^{2} / c^{4}$ is from the background process of $D^{+} \rightarrow K_{L}^{0} \pi^{+}$, where the $K_{L}^{0}$ is undetected and the charged pion is mis-identified as muon. After subtracting the backgrounds, $409.0 \pm 21.2$ signal events were retained. After correcting for the reconstruction efficiency, the branching fraction was measured to be $\mathscr{B}\left(D^{+} \rightarrow \mu^{+} v_{\mu}\right)=\left(3.71 \pm 0.19_{\text {stat }} \pm 0.06_{\text {syst }}\right) \times 10^{-4}$, which is the most precise 
measurement to date. Combining this branching fraction measurement and the Particle Data Group (PDG) values of $D^{+}$lifetime, $M_{D}, m_{\mu}$ and magnitude of $V_{c d}$ determined from the global SM fit, the decay constant is determined to be $f_{D}=\left(203.2 \pm 5.3_{\text {stat }} \pm 1.8_{\text {syst }}\right) \mathrm{MeV}$. Alternately, the magnitude of CKM matrix element $V_{c d}$ is extracted to be $\left|V_{c d}\right|=0.2210 \pm 0.0058_{\text {stat }} \pm 0.0047_{\text {syst }}$ [4].
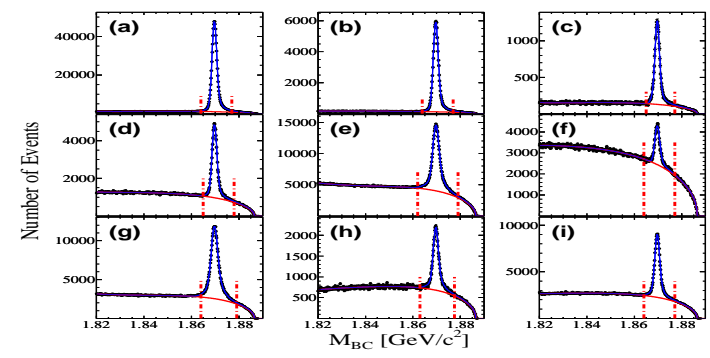

Figure 1: The $\mathrm{M}_{\mathrm{BC}}$ spectra for 9 tag modes (a) $K^{+} \pi^{-} \pi^{-}$, (b) $K_{S}^{0} \pi^{-}$, (c) $K_{S}^{0} K^{-}$, (d) $K^{+} K^{-} \pi^{-}$, (e) $K^{+} \pi^{-} \pi^{-} \pi^{0}$, (f) $\pi^{+} \pi^{-} \pi^{-}$, (g) $K_{S}^{0} \pi^{-} \pi^{0}$, (h) $K^{+} \pi^{-} \pi^{-} \pi^{-} \pi^{+}$and (i) $K_{S}^{0} \pi^{-} \pi^{-} \pi^{+}$.

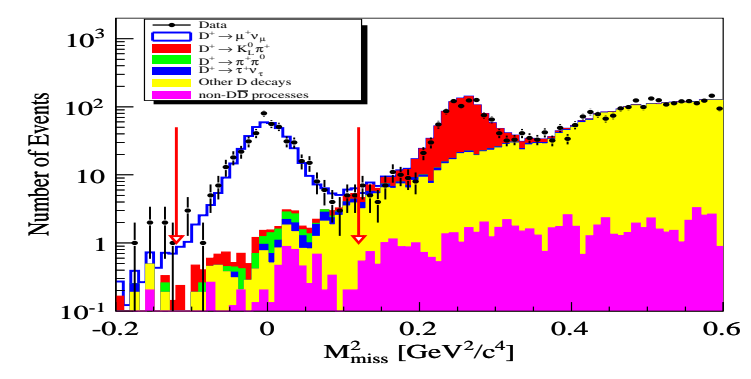

Figure 2: The $\mathrm{M}_{\text {miss }}^{2}$ distribution for the $D^{+} \rightarrow$ $\mu^{+} v_{\mu}$ candidates, where two red arrows denote the signal region.

\subsection{Leptonic $D_{s}^{+}$decays}

By analyzing the data taken at $4.009 \mathrm{GeV}$, we also studied the leptonic $D_{s}^{+}$decays [5]. From nine $D_{s}^{-}$hadronic decay modes $K_{S}^{0} K^{-}, K^{+} K^{-} \pi^{-}, K^{+} K^{-} \pi^{-} \pi^{0}, K_{S}^{0} K^{+} \pi^{-} \pi^{-}, \pi^{+} \pi^{-} \pi^{-}, \pi^{-} \eta$, $\pi^{-} \pi^{0} \eta, \pi^{-} \eta^{\prime}\left(\eta^{\prime} \rightarrow \pi^{+} \pi^{-} \eta\right)$ and $\pi^{-} \eta^{\prime}\left(\eta^{\prime} \rightarrow \rho^{0} \gamma\right), 15127 \pm 321$ singly tagged $D_{s}^{-}$mesons were accumulated. In the system recoiling against the single $D_{s}^{-}$tags, the signal events of $D_{s}^{+} \rightarrow \ell^{+} v_{\ell}$ ( $\ell=\mu$, or $\tau$ ) decays were selected by requiring only one charged track with the opposite charge to the $D_{s}^{-}$tag. Analyzing these doubly tagged $D_{s}^{+} D_{s}^{-}$events with constraining the ratio of $\mu^{+} v_{\mu}$ yield to $\tau^{+} v_{\tau}$ yield to the value given by the SM, we obtained $69.3 \pm 9.3 D_{s}^{+} \rightarrow \mu^{+} v_{\mu}$ and $32.5 \pm 4.3 D_{s}^{+} \rightarrow \tau^{+} v_{\tau}$ events. These yield the branching fractions $\mathscr{B}\left(D_{s}^{+} \rightarrow \mu^{+} v_{\mu}\right)=(0.495 \pm$ $\left.0.067_{\text {stat }} \pm 0.026_{\text {syst }}\right) \%, \mathscr{B}\left(D_{s}^{+} \rightarrow \tau^{+} v_{\tau}\right)=\left(4.83 \pm 0.65_{\text {stat }} \pm 0.26_{\text {syst }}\right) \%$, and the decay constant $f_{D_{s}}=\left(241.0 \pm 16.3_{\text {stat }} \pm 6.6_{\text {syst }}\right) \mathrm{MeV}[5]$.

\section{Semileptonic $D \rightarrow P \ell v(\mathbf{P}=$ pseudoscalar meson) decays}

In the SM, neglecting the lepton mass, the differential decay rate for $D^{+} \rightarrow P e^{+} v_{e}\left(P=K^{-}\right.$, $\pi^{-}, \bar{K}^{0}$ or $\pi^{0}$ ) is given by

$$
\frac{d \Gamma}{d q^{2}}=X \frac{G_{F}^{2}}{24 \pi^{3}}\left|V_{c s(d)}\right|^{2} p^{3}\left|f_{+}^{P}\left(q^{2}\right)\right|^{2}
$$

where $p$ is the momentum of the pseudoscalar meson $P$ in the rest frame of the $D$ meson, $q$ is the four momentum transfer, i.e., the invariant mass of the electron and neutrino system, and $f_{+}^{P}\left(q^{2}\right)$ is the form factor which describes the strong interaction between the final state quarks and is usually parameterized in data analysis. In Eq. (4.1), $X$ is a multiplicative factor due to isospin, which equals to 1 for modes $D^{0} \rightarrow K^{-} e^{+} v_{e}, D^{0} \rightarrow \pi^{-} e^{+} v_{e}, D^{+} \rightarrow \bar{K}^{0} e^{+} v_{e}$ and $1 / 2$ for mode $D^{+} \rightarrow \pi^{0} e^{+} v_{e}$. Similar to leptonic $D_{(s)}^{+}$decays, by measuring the semileptonic $D$ decay rates, these decays also provide an excellent chance to validate the lattice QCD calculations of $f_{+}^{P}\left(q^{2}\right)$, and to determine the CKM elements $\left|V_{c s}\right|$ and $\left|V_{c d}\right|$. 


\section{1 $D^{0} \rightarrow K^{-} e^{+} v_{e}$ and $D^{0} \rightarrow \pi^{-} e^{+} v_{e}$}

The dynamics of the $D^{0} \rightarrow K^{-} e^{+} v_{e}$ and $D^{0} \rightarrow \pi^{-} e^{+} v_{e}$ decays were well studied at the BESIII experiment. Similar to the analysis of $D^{+} \rightarrow \mu^{+} v$ decays, we first selected single $\bar{D}^{0}$ tags from $e^{+} e^{-} \rightarrow D^{0} \bar{D}^{0}$ events in the data taken at $3.773 \mathrm{GeV}$, then selected the events for $D^{0} \rightarrow K^{-} e^{+} v_{e}$ and $D^{0} \rightarrow \pi^{-} e^{+} v_{e}$ decays in the system recoiling against the $\bar{D}^{0}$ tags. From five $\bar{D}^{0}$ hadronic decay modes $K^{+} \pi^{-}, K^{+} \pi^{-} \pi^{0}, K^{+} \pi^{+} \pi^{-} \pi^{-}, K^{+} \pi^{+} \pi^{-} \pi^{-} \pi^{0}$ and $K^{+} \pi^{-} \pi^{0} \pi^{0}$, we accumulated the single $\bar{D}^{0}$ tags. Figure 3 shows beam energy-constraint mass spectrum for each of these 5 modes, from which we obtained $(279.33 \pm 0.37) \times 10^{4}$ single $\bar{D}^{0}$ tags. In the recoil side of these single $\bar{D}^{0}$ tags we selected these two semileptonic decays. Figure 4 shows the $\mathrm{U}_{\text {miss }}$ distributions of the candidates for these two decays. From these $\mathrm{U}_{\text {miss }}$ distributions we observed $70727 \pm 278$ and $6297 \pm 87$ signal events for $D^{0} \rightarrow K^{-} e^{+} v_{e}$ and $D^{0} \rightarrow \pi^{-} e^{+} v_{e}$ decays, respectively. With these numbers of the single $\bar{D}^{0}$ tags and the double $D^{0} \bar{D}^{0}$ tags as well as corresponding efficiencies we measured the branching fractions $\mathscr{B}\left(D^{0} \rightarrow K^{-} e^{+} v_{e}\right)=\left(3.505 \pm 0.014_{\text {stat }} \pm 0.033_{\text {syst }}\right) \%$ and $\mathscr{B}\left(D^{0} \rightarrow \pi^{-} e^{+} v_{e}\right)=\left(0.295 \pm 0.004_{\text {stat }} \pm 0.003_{\text {syst }}\right) \%$ [6], which are the most precise measurements in the world to date.

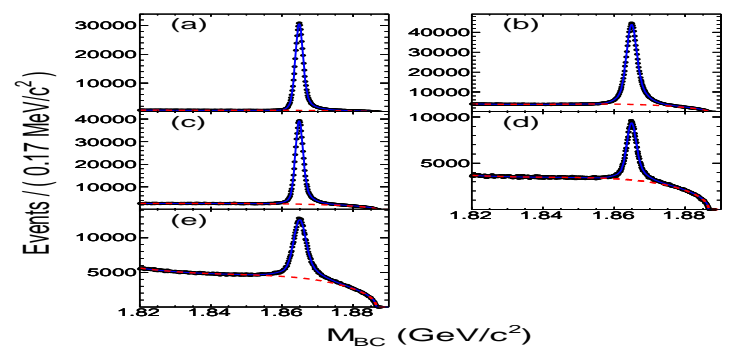

Figure 3: The $\mathrm{M}_{\mathrm{BC}}$ spectra for 5 tag modes (a) $K^{+} \pi^{-}$, (b) $K^{+} \pi^{-} \pi^{0}$, (c) $K^{+} \pi^{+} \pi^{-} \pi^{-}$, (d) $K^{+} \pi^{+} \pi^{-} \pi^{-} \pi^{0},(\mathrm{e}) K^{+} \pi^{-} \pi^{0} \pi^{0}$.

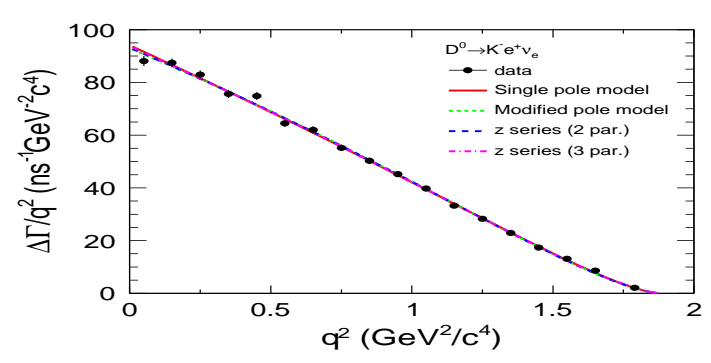

Figure 5: The differential decay rates for $D^{0} \rightarrow$ $K^{-} e^{+} v_{e}$ with the fit results overlaid.

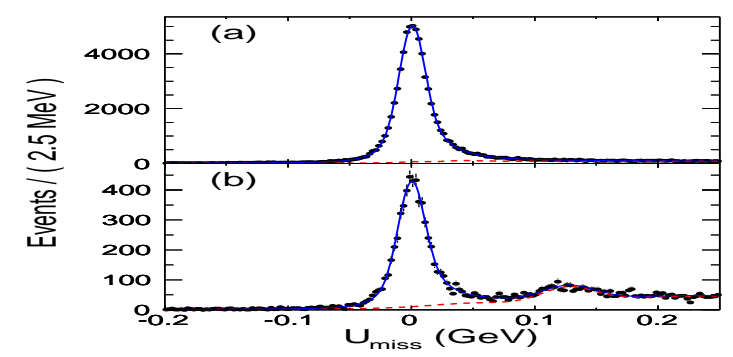

Figure 4: The $\mathrm{U}_{\text {miss }}$ distribution for (a) $D^{0} \rightarrow$ $K^{-} e^{+} v_{e}$ and (b) $D^{0} \rightarrow \pi^{-} e^{+} v_{e}$ candidates.

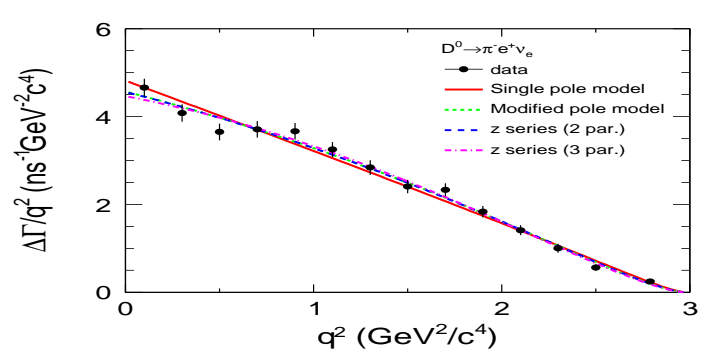

Figure 6: The differential decay rates for $D^{0} \rightarrow$ $\pi^{-} e^{+} v_{e}$ with the fit results overlaid.

To determine the form factors $f^{K}\left(q^{2}\right)$ and $f^{\pi}\left(q^{2}\right)$ as well as CKM elements $\left|V_{\mathrm{cs}}\right|$ and $\left|V_{\mathrm{cd}}\right|$ we measured differential rates of these two decays in 18 or $14 q^{2}$ bins as shown in Fig. 5 and Fig. 6. Using the expected differential decay rates given in Eq. (4.1) and the form factor parameterized with two-parameter $z$ series expansion to fit the measured differential decay rates, we directly measured the products $f_{+}^{K}(0)\left|V_{c s}\right|=0.7172 \pm 0.0025 \pm 0.0035$ and $f_{+}^{\pi}(0)\left|V_{c d}\right|=0.1345 \pm 0.0018 \pm$ 
0.0009 as well as form the factor parameters [6]. Using the measured values for these products in conjunction with the values for $\left|V_{c s}\right|$ and $\left|V_{c d}\right|$ determined from a global SM fit, we determined $f_{+}^{K}(0)=0.7368 \pm 0.0026_{\text {stat }} \pm 0.0036_{\text {syst }}$ and $f_{+}^{\pi}(0)=0.6372 \pm 0.0080_{\text {stat }} \pm 0.0044_{\text {syst }}[6]$.

4.2 $D^{+} \rightarrow \bar{K}^{0} e^{+} v_{e}$ and $D^{+} \rightarrow \pi^{0} e^{+} v_{e}$

In the system recoiling against the $(170.31 \pm 0.34) \times 10^{4}$ single $D^{-}$tags as these used in study of $D^{+} \rightarrow \mu^{+} v$ decays mentioned above [4], we selected $D^{+} \rightarrow \bar{K}^{0} e^{+} v_{e}$ and $D^{+} \rightarrow \pi^{0} e^{+} v_{e}$ events, where the $\bar{K}^{0}$ and $\pi^{0}$ mesons were reconstructed with $\pi^{+} \pi^{-}$and $\gamma \gamma$, respectively. We totally observed $26008 \pm 168 D^{+} \rightarrow \bar{K}^{0} e^{+} v_{e}$ and $3402 \pm 70 D^{+} \rightarrow \pi^{0} e^{+} v_{e}$ signal events, and measured branching fractions $\mathscr{B}\left(D^{+} \rightarrow \bar{K}^{0} e^{+} v_{e}\right)=\left(8.604 \pm 0.056_{\text {stat }} \pm 0.151_{\text {syst }}\right) \%$ and $\mathscr{B}\left(D^{+} \rightarrow \pi^{0} e^{+} v_{e}\right)=$ $\left(0.3631 \pm 0.0075_{\text {stat }} \pm 0.0051_{\text {syst }}\right) \%$. Using these branching fractions and branching fractions for $D^{0} \rightarrow K^{-} e^{+} v_{e}$ and $D^{0} \rightarrow \pi^{-} e^{+} v_{e}$ decays presented in Section 4.1, as well as the lifetimes of $D^{0}$ and $D^{+}$mesons [7], we obtained the ratios $I_{K} \equiv \frac{\Gamma\left(D^{0} \rightarrow K^{-} e^{+} v_{e}\right)}{\Gamma\left(D^{+} \rightarrow \bar{K}^{0} e^{+} v_{e}\right)}=1.03 \pm 0.01 \pm 0.02$ and $I_{\pi} \equiv$ $\frac{\Gamma\left(D^{0} \rightarrow \pi^{-} e^{+} v_{e}\right)}{2 \Gamma\left(D^{+} \rightarrow \pi^{0} e^{+} v_{e}\right)}=1.03 \pm 0.03 \pm 0.02$, which are consistent with isospin symmetry.

We also analyzed differential decay rates for these two semileptonc $D^{+}$decays and measured the products $f_{+}^{K}(0)\left|V_{c s}\right|=0.7053 \pm 0.0040 \pm 0.0012$ and $f_{+}^{\pi}(0)\left|V_{c s}\right|=0.1400 \pm 0.0026 \pm 0.0007$. Using these values in conjunction with $\left|V_{c s(d)}\right|$ obtained from global SM fit [7] we determined $f_{+}^{K}(0)=0.725 \pm 0.004 \pm 0.012$ and $f_{+}^{\pi}(0)=0.622 \pm 0.012 \pm 0.004$. Alternatively, using these values of the products together with LQCD calculations of the form factors $f_{+}^{K}(0)=0.7470 \pm$ $0.0186[8]$ and $f_{+}^{\pi}(0)=0.666 \pm 0.029$ [9] we determined the CKM elements $\left|V_{\mathrm{cs}}\right|=0.944 \pm$ $0.005_{\text {stat }} \pm 0.015_{\text {syst }} \pm 0.024_{\mathrm{LQCD}}$ and $\left|V_{\text {cd }}\right|=0.210 \pm 0.024_{\text {stat }} \pm 0.001_{\text {syst }} \pm 0.009_{\mathrm{LQCD}}$.

4.3 $D^{+} \rightarrow \bar{K}^{0} e^{+} v_{e}$ with $\bar{K}^{0} \rightarrow \pi^{0} \pi^{0}$

With $\sim 1.5$ millions of singly tagged $D^{-}$sample accumulated from six hadronic decay modes of $D^{-}$meson we investigated the semileptonic $D^{+} \rightarrow \bar{K}^{0} e^{+} v_{e}$ decay, where $\bar{K}^{0}$ was reconstructed with $\bar{K}^{0} \rightarrow \pi^{0} \pi^{0}$. We observed a total of $5013 \pm 78$ signal events for this semileptonic decays and measured the branching fraction $\mathscr{B}\left(D^{+} \rightarrow \bar{K}^{0} e^{+} v_{e}\right)=\left(8.59 \pm 0.014_{\text {stat }} \pm 0.21_{\text {syst }}\right) \%$ [10].

$4.4 D^{+} \rightarrow \bar{K}^{0} \mu^{+} v_{\mu}$

In the recoil side of $\sim 1.5$ millions of singly tagged $D^{-}$mesons we studied the semileptonic $D^{+} \rightarrow \bar{K}^{0} \mu^{+} v_{\mu}$ decays, where the $\bar{K}^{0}$ meson was reconstructed from decay processes of $\bar{K}^{0} \rightarrow$ $\bar{K}_{s}^{0} \rightarrow \pi^{+} \pi^{-}$and $\bar{K}^{0} \rightarrow \bar{K}_{s}^{0} \rightarrow \pi^{0} \pi^{0}$. We observed $16516 \pm 130$ and $4198 \pm 33$ signal events for $D^{+} \rightarrow \bar{K}^{0} \mu^{+} v_{\mu}$ with $\bar{K}^{0} \rightarrow \pi^{+} \pi^{-}$and $\bar{K}^{0} \rightarrow \pi^{0} \pi^{0}$, respectively. With these numbers of signal events together with the 1.5 millions of the singly tagged $D^{-}$mesons, we measured the branching fraction $\mathscr{B}\left(D^{+} \rightarrow \bar{K}^{0} \mu^{+} v_{\mu}\right)=\left(8.72 \pm 0.07_{\text {stat }} \pm 0.18_{\text {syst }}\right) \%$ [11].

4.5 $D^{+} \rightarrow K_{L}^{0} e^{+} v_{e}$

Using a very similar single $D^{-}$tag sample as that used in study of $D^{+} \rightarrow \mu^{+} v_{\mu}$ mentioned above and adopting a technique of partial reconstructing $K_{L}^{0}$ meson, we studied the semileptonic decay $D^{+} \rightarrow K_{L}^{0} e^{+} v_{e}$ [12], and measured branching fraction $\mathscr{B}\left(D^{+} \rightarrow K_{L}^{0} e^{+} v_{e}\right)=(4.482 \pm$ $0.027 \pm 0.103) \%$, as well as the $C P$ asymmetry of $D^{+} \rightarrow K_{L}^{0} e^{+} v_{e}$ and $D^{-} \rightarrow K_{L}^{0} e^{-} \bar{v}_{e}$ decays, $\mathscr{A}_{C P}=\left(\mathscr{B}\left(D^{+} \rightarrow K_{L}^{0} e^{+} v_{e}\right)-\mathscr{B}\left(D^{+} \rightarrow K_{L}^{0} e^{-} \bar{v}_{e}\right)\right) /\left(\mathscr{B}\left(D^{+} \rightarrow K_{L}^{0} e^{+} v_{e}\right)+\mathscr{B}\left(D^{+} \rightarrow K_{L}^{0} e^{-} \bar{v}_{e}\right)\right)=$ 
$(-0.59 \pm 0.60 \pm 1.48) \%$. We also measured the product of $f_{+}^{K}(0)$ times $\left|V_{c s}\right|$, and alternatively determined $f_{+}^{K}(0)$ and $\left|V_{c s}\right|$. These are $f_{+}^{K}(0)\left|V_{c s}\right|=0.728 \pm 0.006_{\text {stat }} \pm 0.011_{\text {sys }}, f_{+}^{K}(0)=$ $0.748 \pm 0.007_{\text {stat }} \pm 0.012_{\text {sys }}$, and $\left|V_{c s}\right|=0.975 \pm 0.008_{\text {stat }} \pm 0.015_{\text {sys }} \pm 0.025_{\mathrm{LQCD}}$.

\section{Semileptonic $D \rightarrow \mathscr{V} e^{+} v_{e}(\mathscr{V}=$ vector meson $)$ decays}

The differential rate of the semileptonic $D^{+} \rightarrow \mathscr{V} e^{+} v_{e}$ decay is described by five variables. Taking $\mathscr{V}=K^{* 0}$ as an example, these five variables are the invariant mass $m_{K \pi}$ of $K$ and $\pi$ system, the four momentum transfer squared $q^{2}$, the angle $\theta_{K}$ between the $K$ and the $D$ direction in the $K^{-} \pi^{+}$rest frame, the angle $\theta_{e}$ between the $v_{e}$ and the $D$ direction in the $e^{+} v_{e}$ rest frame, and the acoplanarity angle $\chi$ between the two decay planes $[13,14]$. This decay rate is given by $[13,14]$

$$
\frac{d \Gamma\left(D \rightarrow K^{* 0} e^{+} v_{e}\right)}{d m_{K \pi}^{2} d q^{2} d \cos \theta_{K} d \cos \theta_{e} d \chi}=\frac{G_{F}^{2}\left|V_{C s}\right|^{2}}{(4 \pi)^{6} m_{D}^{2}} X \beta I\left(m_{K \pi}^{2}, q^{2}, \theta_{K}, \theta_{e}, \chi\right),
$$

where $X=p_{K \pi} m_{D}, p_{K \pi}$ is the momentum of the $K \pi$ system in the $D$ rest frame, $m_{D}$ is the mass of the $D$ meson, $\beta=2 p^{*} / m, p^{*}$ is the breakup momentum of the $K \pi$ system in the $D$ rest frame, and $I$ can be expressed in terms of helicity amplitudes $H_{0, \pm}$ :

$$
H_{0}\left(q^{2}\right)=\frac{1}{2 m_{K \pi} q}\left[\left(m_{D}^{2}-m_{K \pi}^{2}-q^{2}\right)\left(m_{D}+m_{K \pi}\right) A_{1}\left(q^{2}\right)-4 \frac{m_{D}^{2} p_{K \pi}^{2}}{m_{D}+m_{K \pi}} A_{2}\left(q^{2}\right)\right]
$$

and

$$
H_{ \pm}\left(q^{2}\right)=\left(m_{D}+m_{K \pi}\right) A_{1}\left(q^{2}\right) \mp \frac{2 m_{D} p_{K \pi}}{m_{D}+m_{K \pi}} V\left(q^{2}\right) .
$$

In these three helicity amplitudes, $A_{1}\left(q^{2}\right)$ and $A_{2}\left(q^{2}\right)$ are two axial-vector form factors, and $V\left(q^{2}\right)$ is the vector form factor. Under assumption of the single pole dominance, these form factors are given by

$$
A_{1}\left(q^{2}\right)=\frac{A_{1}(0)}{1-q^{2} / m_{A}^{2}}, \quad A_{2}\left(q^{2}\right)=\frac{A_{2}(0)}{1-q^{2} / m_{A}^{2}} \quad \text { and } \quad V\left(q^{2}\right)=\frac{V(0)}{1-q^{2} / m_{V}^{2}},
$$

where $m_{A}$ and $m_{V}$ are the axial-vector and vector pole masses, respectively.

$5.1 D^{+} \rightarrow K^{-} \pi^{+} e^{+} v_{e}$

Using a very similar sample of the single $D^{-}$tags as that mentioned in Section 3.1 we studied the semileptonic $D^{+} \rightarrow K^{-} \pi^{+} e^{+} v_{e}$ decays. By studying the $\mathrm{U}_{\text {miss }}$ of the single $D^{-}$tags and the $K^{-} \pi^{+} e^{+}$in the recoil side of the single $D^{-}$tags, we observed 18262 signal events for $D^{+} \rightarrow$ $K^{-} \pi^{+} e^{+} v_{e}$ decay in the system recoiling against these single $D^{-}$tags, and measured branching fraction $\mathscr{B}\left(D^{+} \rightarrow K^{-} \pi^{+} e^{+} v_{e}\right)=\left(3.71 \pm 0.03_{\text {stat }} \pm 0.08_{\text {sys }}\right) \%$. To investigate the dynamic of these decays, we studied the five kinematic variables as defined in the Section 5 for these decays. Figure 7 shows distributions of these five kinematic variables determined from these selected events. A partial wave analysis was performed on these selected events and showed that the dominant $\bar{K}^{* 0}$ component is companied by a S-wave contribution with a fraction of $(6.05 \pm 0.22 \pm 0.18) \%$ of the total rate, while other components can be negligible. From this analysis we obtained the mass and the total width of $\bar{K}^{* 0}(892)$ to be $M_{\bar{K}^{* 0}(892)}=(894.60 \pm 0.25 \pm 0.08) \mathrm{MeV}$ and $\Gamma_{\bar{K}^{* 0}(892)}=(46.42 \pm$ 

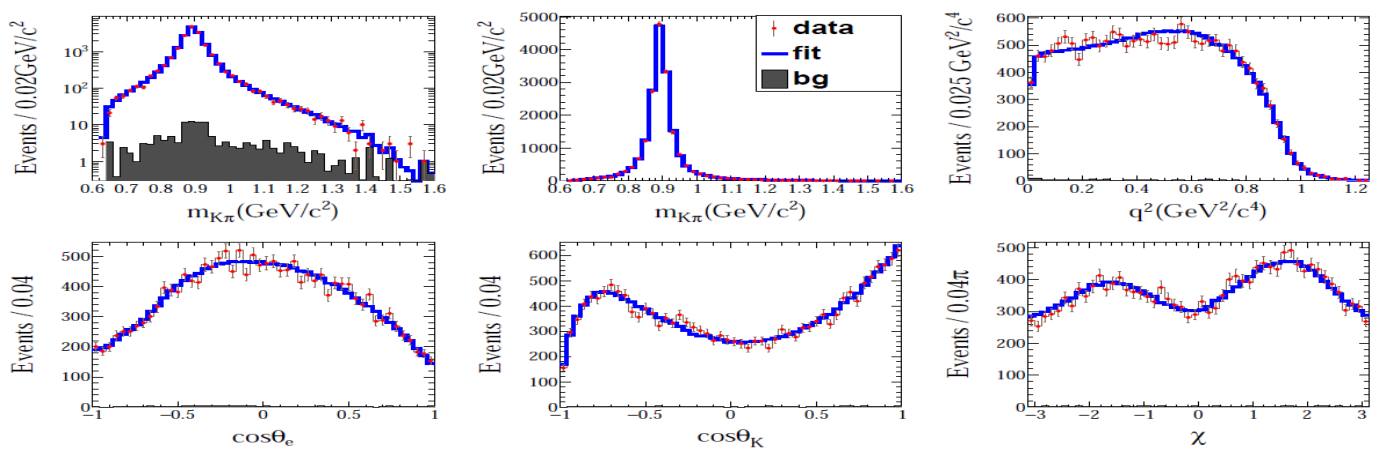

Figure 7: Distributions of the kinematic variables of the $D^{+} \rightarrow K^{-} \pi^{+} e^{+} v_{e}$.

$0.56 \pm 0.15) \mathrm{MeV}$. From this analysis we obtained the form factor $A_{1}(0)=0.585 \pm 0.011 \pm 0.017$ the form factor ratio $r_{V}=\frac{V(0)}{A_{1}(0)}=1.411 \pm 0.058 \pm 0.007$ and $r_{2}=\frac{V_{2}(0)}{A_{1}(0)}=0.788 \pm 0.042 \pm 0.008$. In addition, we measured the vector pole mass $m_{V}=\left(1.81_{-0.17}^{+0.25} \pm 0.02\right) \mathrm{MeV} / \mathrm{c}^{2}$ and the axial-vector pole mass $m_{A}=\left(2.61_{-0.17}^{+0.22} \pm 0.03\right) \mathrm{MeV} / \mathrm{c}^{2}[15]$.

$5.2 D^{+} \rightarrow \omega e^{+} v_{e}$ and $D^{+} \rightarrow \phi e^{+} v_{e}$

Similarly, we also studied the $D^{+} \rightarrow \omega e^{+} v_{e}$ decay, where $\omega \rightarrow \pi^{+} \pi^{-} \pi^{0}$. In the recoil side of 1.58 million of singly tagged $D^{-}$sample we observed $491 \pm 32 D^{+} \rightarrow \omega e^{+} v_{e}$ decays, and measured branching fraction $\mathscr{B}\left(D^{+} \rightarrow \omega e^{+} v_{e}\right)=(0.163 \pm 0.011 \pm 0.008) \%$ [16]. By analyzing multi-dimensional distributions of the five kinematic variables determined from our selected sample of $D^{+} \rightarrow \omega e^{+} v_{e}$ decays, we measured the form factor ratios $r_{V}=\frac{V(0)}{A_{1}(0)}=1.24 \pm 0.09 \pm 0.06$ and $r_{2}=\frac{V_{2}(0)}{A_{1}(0)}=1.06 \pm 0.15 \pm 0.05$.

In this singly tagged $D^{-}$sample we searched for $D^{+} \rightarrow \phi e^{+} v_{e}$ decay. But, we did not observe any significant signal event for this decay. We set an upper limit of the decay branching fraction to be $\mathscr{B}\left(D^{+} \rightarrow \phi e^{+} v_{e}\right)<1.3 \times 10^{-5}$ at $90 \% \mathrm{CL}$.

\section{Semileptonic $\Lambda_{c}^{+}$decay and rare $D$ decays}

From the $0.57 \mathrm{fb}^{-1}$ of data taken at $\sqrt{s}=4.599 \mathrm{GeV}$ we selected $(1.4 \pm 0.2) \times 10^{4}$ singly tagged $\bar{\Lambda}_{c}^{-}$baryons. In the system recoiling against these singly tagged $\bar{\Lambda}_{c}^{-}$baryons we observed $(109.4 \pm 10.9) \Lambda_{c}^{+} \rightarrow \Lambda e^{+} v_{e}$ decay events, and measured branching fraction $\mathscr{B}\left(\Lambda_{c}^{+} \rightarrow \Lambda e^{+} v_{e}\right)=$ (3.63 $\pm 0.38 \pm 0.30) \%$ [17] for the first time. Some theoretical model predictions for this branching fraction vary in a range from $1.4 \% \sim 9.4 \%$. Thus our experimental result provides a stringent test of these models.

The SM predicts that the branching fraction for $D^{+} \rightarrow K(\pi)^{+} e^{+} e^{-}$decays are in the range from $10^{-8}$ to $10^{-6}$. However some new physics effects may enhance these decay rates, resulting in these branching fractions at the level of $10^{-5}$ to $10^{-6}$. The lepton number volition decay $D^{+} \rightarrow$ $K(\pi)^{-} e^{+} e^{+}$is strictly forbidden in the SM. So searching for these decays supply a way to search for NP beyond the SM. In the full data sample taken at $\sqrt{s}=3.773 \mathrm{GeV}$ we searched for some rare $D^{+}$decays with reconstructed $D^{+}$meson decays to these semileptonic final states. But we did not observe any evdence for these decays. As preliminary results we set upper limits on these 
rare semileptonic $D^{+}$decay branching fractions to be $\mathscr{B}\left(D^{+} \rightarrow K^{+} e^{+} e^{-}\right)<1.2 \times 10^{-6}, \mathscr{B}\left(D^{+} \rightarrow\right.$ $\left.\pi^{+} e^{+} e^{-}\right)<0.3 \times 10^{-6}, \mathscr{B}\left(D^{+} \rightarrow K^{-} e^{+} e^{+}\right)<0.6 \times 10^{-6}$ and $\mathscr{B}\left(D^{+} \rightarrow \pi^{-} e^{+} e^{+}\right)<1.2 \times 10^{-6}$ at $90 \%$ C.L..

\section{Validate theoretical calculations of $f_{+}^{K}\left(q^{2}\right), f_{+}^{\pi}\left(q^{2}\right)$ and $f_{D^{+}}$}

\subsection{Comparison of measured $f_{+}^{K}\left(q^{2}\right)$ and $f_{+}^{\pi}\left(q^{2}\right)$ withe those calculated in LQCD}

Figure 8 and Fig. 9 show comparisons between our measured form factor shapes [6] and those calculated in LQCD [18] for $D^{0} \rightarrow K^{-} e^{+} v_{e}$ and $D^{0} \rightarrow \pi^{-} e^{+} v_{e}$ decays, respectively. From these two figures we find that, although our measured values of $f_{+}^{K}\left(q^{2}\right)$ and $f_{+}^{\pi}\left(q^{2}\right)$ are consistent within uncertainties with those calculated in LQCD, the measured values of the form factors significantly deviate from the most probable values calculated in LQCD in the regions above $0.75 \mathrm{GeV}^{2} / c^{4}$ and $1.5 \mathrm{GeV}^{2} / c^{4}$ for $D^{0} \rightarrow K^{-} e^{+} v_{e}$ and $D^{0} \rightarrow \pi^{-} e^{+} v_{e}$ decays, respectively. The precision of our measured $f_{+}^{K}\left(q^{2}\right)$ and $f_{+}^{\pi}\left(q^{2}\right)$ is higher than that of the LQCD calculations by a factor of $3 \sim 4$.

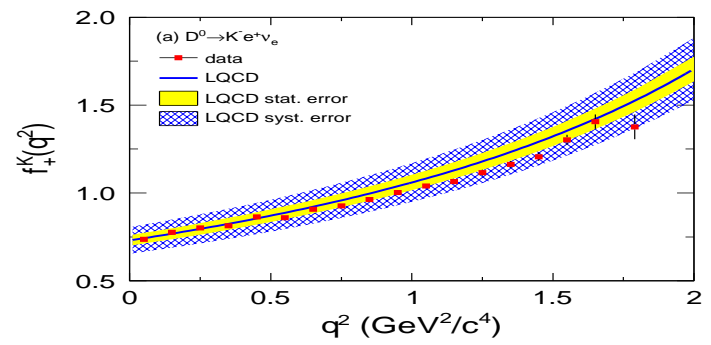

Figure 8: Comparison between the measured form factor at the BES-III and that calculated in LQCD for $D^{0} \rightarrow K^{-} e^{+} v_{e}$ decays.

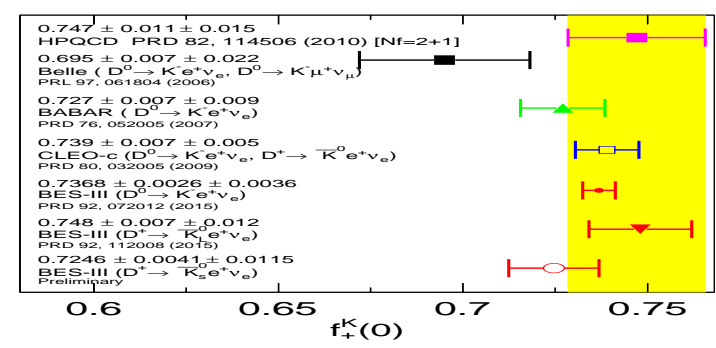

Figure 10: Comparison between measured form factor $f_{+}^{K}(0)$ and that calculated in LQCD.

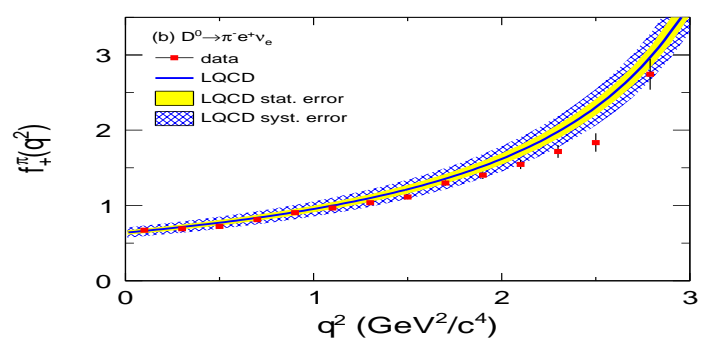

Figure 9: Comparison between the measured form factor at the BES-III and that calculated in LQCD for $D^{0} \rightarrow \pi^{-} e^{+} v_{e}$ decays.

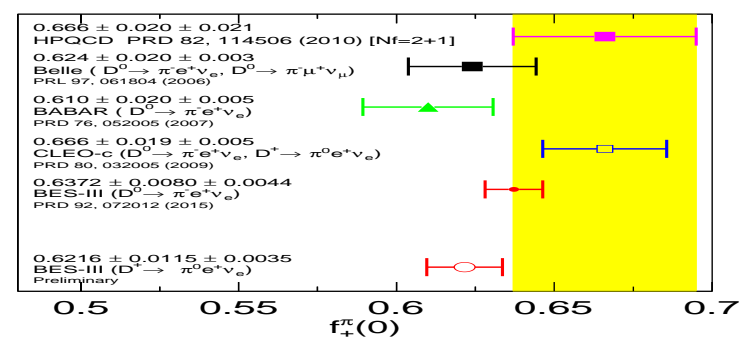

Figure 11: Comparison between measured form factor $f_{+}^{\pi}(0)$ and that calculated in LQCD.

Figure 10 and Fig. 11 show comparisons between our measured form factors at $q^{2}=0$ and these measured at other experiments together with that calculated in LQCD. Our measured form factors are consistent with those measured at the BABAR, Belle and CLEO-c experiments, but the precisions of our measured form factors from $D^{0} \rightarrow K^{-} e^{+} v_{e}$ and $D^{0} \rightarrow \pi^{-} e^{+} v_{e}$ decays are more higher than others. In addition, the precisions of our measured form factors at $q^{2}=0$ from these two decays are also higher than that calculated in LQCD by a factor of $3 \sim 4$, and thereby our 
precisely measured values for $f_{+}^{K}(0)$ and $f_{+}^{\pi}(0)$ will allow incisive tests of any further theoretical calculations of these form factors.

\subsection{Comparison of measured $f_{D^{+}}$with theoretical predictions}

Figure 12 shows comparison between our measured $f_{D^{+}}$and those measured at other experiments together with theoretical calculations of the decay constant. Our measured $f_{D^{+}}$is consistent with those measured at other experiments, but with the best precision. Our measured value of $f_{D^{+}}=(203.91 \pm 5.72 \pm 1.97) \mathrm{MeV}$ is lower than the most precise value of $f_{D^{+}}=\left(212.6 \pm 0.4_{-1.2}^{+1.0}\right)$ $\mathrm{MeV}$ calculated in LQCD by $1.4 \sigma$.

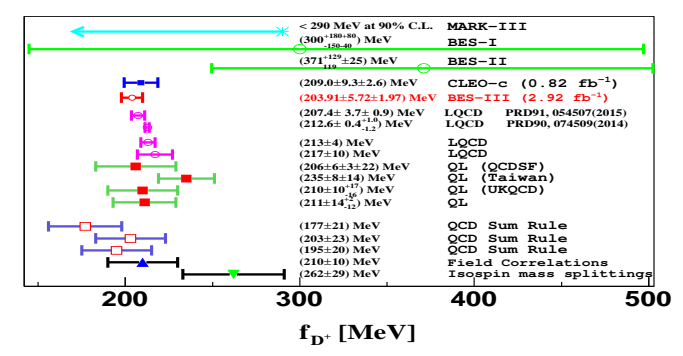

Figure 12: Comparison of measured values for $f_{D^{+}}$ with those of theoretical calculations.

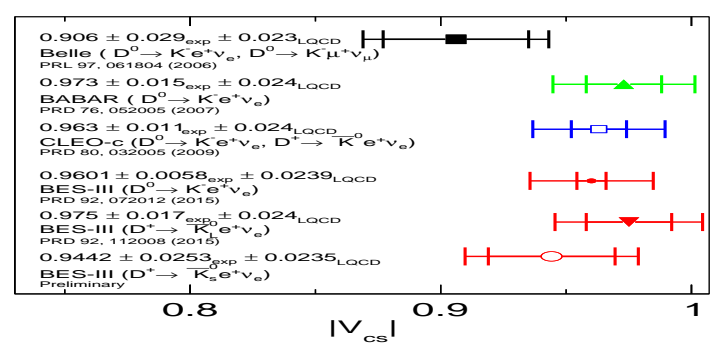

Figure 13: Comparison of $\left|V_{\mathrm{cs}}\right|$ measured at $B A B A R$, Belle, CLEO-c and BES-III experiments.

\section{Status of $\left|V_{\mathrm{cs}}\right|,\left|V_{\mathrm{cd}}\right|$ and test of three-generation unitarity}

Since the CKM elements are fundamental parameters of the SM, they should be measured as precisely as possible. Figure 13 and Fig. 14 show comparisons of our determined $\left|V_{\mathrm{cs}}\right|$ and $\left|V_{\mathrm{cd}}\right|$ with those determined using $f_{+}^{K(\pi)}(0)\left|V_{\mathrm{cs}(\mathrm{d})}\right|$ which were measured at the BABAR, Belle and CLEOc experiments in conjunctions with $f_{+}^{K}(0)=0.7470 \pm 0.0186$ [8] and $f_{+}^{\pi}(0)=0.666 \pm 0.029$ [9]. In these determined values listed on these two figures, the first errors are the combining both the experimentally statistical and systematic uncertainties, and the second are due to the uncertainties of the LQCD calculations of the form factors. From these one can find that, although we only have 3.56 times larger data than CLEO-c data taken at $3.773 \mathrm{GeV}$ our experimental precisions of the values for $\left|V_{\mathrm{cs}}\right|$ and $\left|V_{\mathrm{cd}}\right|$ determined from only $D^{0} \rightarrow K^{-} e^{+} v_{e}$ and $D^{0} \rightarrow \pi^{-} e^{+} v_{e}$ decays are higher than those determined from $D^{0} \rightarrow K^{-} e^{+} v_{e}, D^{+} \rightarrow \bar{K}^{0} e^{+} v_{e}, D^{0} \rightarrow \pi^{-} e^{+} v_{e}$ and $D^{+} \rightarrow \pi^{0} e^{+} v_{e}$ decays at the CLEO-c by a factor of almost 2 .

To search for NP effect, high precision values of $\left|V_{\mathrm{cs}}\right|$ and $\left|V_{\mathrm{cd}}\right|$ are urgently needed since these could be used to test the three-generation unitarity, and thereby also maximizing the sensitivity of quark flavor physics to NP. In the last few years many authors used our preliminary results on measurements of $D^{+} \rightarrow \mu^{+} v_{\mu}, D^{0} \rightarrow K^{-} e^{+} v_{e}$ and $D^{0} \rightarrow \pi^{-} e^{+} v_{e}$ decays to update the values for $\left|V_{\mathrm{cs}}\right|$ and $\left|V_{\mathrm{cd}}\right|$, and then checked the unitarity of the CKM matrix. Among these a global analysis of all available charm data including our preliminary results of measurements for these decays yields the best precision values of

$$
\left|V_{\mathrm{cs}}\right|=0.983 \pm 0.011 \quad \text { (from all available charm data in the world [20]) }
$$



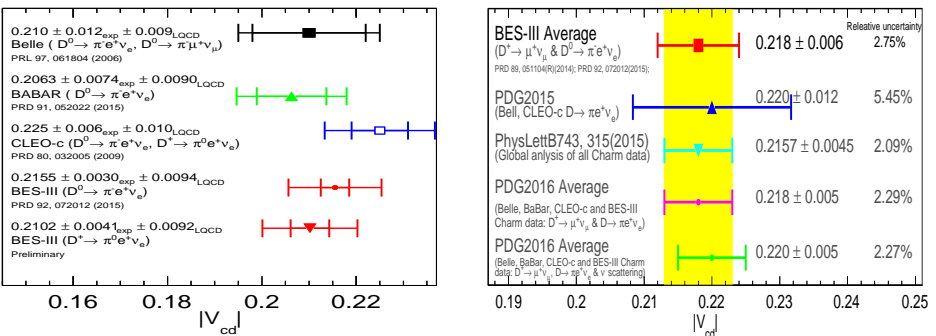

Figure 14: Comparison of $\left|V_{\mathrm{cd}}\right|$ Figure 15: Comparison of $\left|V_{\mathrm{cd}}\right|$ measured at BABAR, Belle, CLEO- (see text).

$\mathrm{c}$ and BES-III experiments.

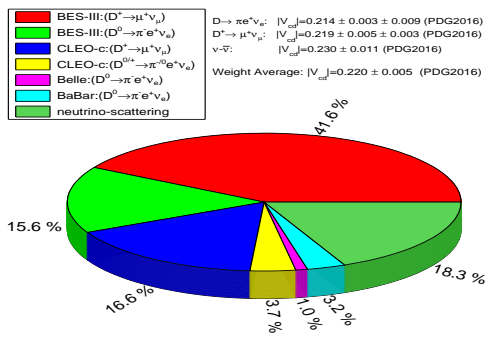

Figure 16: Weight in the weighted average $\left|V_{\text {cd }}\right|$ given in PDG2016.

and

$$
\left|V_{\mathrm{cd}}\right|=0.2157 \pm 0.0045 \quad \text { (from all available charm data in the world [21]) }
$$

With this value for $\left|V_{\text {cd }}\right|$ together with the values for other related CKM elements given in PDG2014 [7], a $2 \sigma$ tension in the first column of the CKM matrix was seen in March 2015 [21]. After adding our measurements of $D^{+} \rightarrow \mu^{+} v_{\mu}$ and $D^{0} \rightarrow \pi^{-} e^{+} v_{e}$ decays together with the CLEO-c measurement of $D^{+} \rightarrow \mu^{+} v_{\mu}$ decay, the PDG issued a new weighted average value

$$
\left|V_{\mathrm{cd}}\right|=0.220 \pm 0.005 \quad(\mathrm{PDG} 2016[22])
$$

in 2016, which improve the precision of the $\left|V_{\mathrm{cd}}\right|$ value by more than $48 \%$. Figure 15 shows comparison of the values for $\left|V_{\text {cd }}\right|$ given in the PDG2015, PDG2016 and that determined by globally analyzing all available charm data in the world [21]. An average for $\left|V_{\mathrm{cd}}\right|$ determined from our measurements of $D^{+} \rightarrow \mu^{+} v_{\mu}$ and $D^{0} \rightarrow \pi^{-} e^{+} v_{e}$ decays at the BES-III is also shown in the Fig. 15. Our measurements contribute more than $57 \%$ weight (see Fig. 16) in each of these two weighted averages for the $\left|V_{\mathrm{cd}}\right|$. With the updated $\left|V_{\mathrm{cd}}\right|=0.220 \pm 0.005$ given in 2016, the PDG found a $2 \sigma$ tension [22] in the second row of the CKM matrix, which in fact confirmed the $2 \sigma$ tension in the first column of the CKM matrix seen in March 2015 [21]. These $2 \sigma$ tensions may imply potential signal for new physics effects. All of these progresses mainly profit from the more precise value for $\left|V_{\mathrm{cd}}\right|$ determined by adding our measurements of $D^{+} \rightarrow \mu^{+} v_{\mu}$ and $D^{0} \rightarrow \pi^{-} e^{+} v_{e}$ decays.

\section{Summary}

Using the data taken with the BES-III detector at the energies near $D \bar{D}, D_{s}^{+} D_{s}^{-}$and $\Lambda_{c}^{+} \bar{\Lambda}_{c}^{-}$production thresholds we measured branching fractions for leptonic and semileptonic charm decays, and investigated the decay dynamic of the $D^{+}$and $D^{0}$ mesons. From the leptonic $D^{+}$and semileptonic $D^{0}$ decays we determined the most precise values for the decay constant $f_{D^{+}}$, the hadronic form factors $f_{+}^{K(\pi)}(0)$, and the form factor shape $f_{+}^{K(\pi)}\left(q^{2}\right)$. These precisely determined quantities have being used to incisively validate the LQCD calculations of these quantities resulting in improving determinations of $B$ decay constants and form factors. When these calculations of the $B$ decay constants and form factors are combined with huge $B$ factory data, precision of the values for $\left|V_{\mathrm{td}}\right|$ and $\left|V_{\mathrm{ub}}\right|$ are improved, which are quantitatively transferred to knowledge of the $B_{d}$ unitarity triangle, and thereby maximizing the sensitivity of quark flavor physics to NP. 
From leptonic $D^{+} \rightarrow \mu^{+} v_{\mu}$ decay we determined $\left|V_{\text {cd }}\right|=0.2210 \pm 0.0058_{\text {stat }} \pm 0.0047_{\text {syst }}$. In addition, from $D^{0} \rightarrow K^{-} e^{+} v_{e}$ and $D^{0} \rightarrow \pi^{-} e^{+} v_{e}$ decays we determined the most experimentally precise values for the CKM elements $\left|V_{\mathrm{cs}}\right|=0.9601 \pm 0.0058_{\exp } \pm 0.0239_{\mathrm{LQCD}}$ and $\left|V_{\mathrm{cd}}\right|=$ $0.2155 \pm 0.006_{\exp } \pm 0.0094_{\mathrm{LQCD}}$. At present, the uncertainties of $\left|V_{\mathrm{cd}}\right|$ and $\left|V_{\mathrm{cs}}\right|$ determined from the semileptonic $D$ decays are still dominated by the uncertainties of $f_{+}^{\pi}(0)$ and $f_{+}^{K}(0)$ calculated in LQCD. If these uncertainties of $f_{+}^{\pi}(0)$ and $f_{+}^{K}(0)$ calculated in LQCD could be negligible, the relative accuracy of $\left|V_{\mathrm{cs}}\right|$ and $\left|V_{\mathrm{cd}}\right|$ determined at the BES-III experiment could reach to $\Delta\left|V_{\mathrm{cs}}\right| /\left|V_{\mathrm{cs}}\right| \sim 0.5 \%$ and $\Delta\left|V_{\mathrm{cd}}\right| /\left|V_{\mathrm{cd}}\right| \sim 1.2 \%$, with which the three-generation unitarity in the first and the second columns as well as the second row of the CKM matrix can be more precisely checked, and thereby these also maximize the sensitivity of quark flavor physics to NP.

Our measurements of form factors for $D \rightarrow \mathscr{V} e^{+} v_{e}$ decays and new measurements on the char$\mathrm{m}$ decay rates provide new experimental data for understanding of these charm decay dynamics.

\section{References}

[1] G. Rong, "Physics program of open charm and heavy $c \bar{c}$ states at the BES-III experiment", Chin. Phys. C 34, 788 (2010).

[2] G. Rong, "Review and Report on Results of Leptonic Decays of $D^{+}$and $D_{s}^{+}$Mesons", Charm2012, Honolulu, Hawaii, May 14-17, 2012, arXiv:1209.0085v1 [hep-ex].

[3] J.Z. Bai et al. (BES Collaboration), Phys. Lett. B 610, 183 (2005).

[4] M. Ablikim et al. (BESIII Collaboration), Phys. Rev. D 89, 051104(R) (2014).

[5] M. Ablikim et al. (BESIII Collaboration), Phys. Rev. D 94, 072004(2016).

[6] M. Ablikim et al. (BESIII Collaboration), Phys. Rev. D 92, 072012 (2015).

[7] K. A. Olive et al. (Particle Data Group), Chin. Phys. C 38, 090001 (2014).

[8] H. Na et al. (HPQCD Collaboration), Phys. Rev. D 82, 114506 (2010).

[9] H. Na et al. (HPQCD Collaboration), Phys. Rev. D 84, 114505 (2011); arXiv: 1305.1462 [hep-lat].

[10] M. Ablikim et al. (BESIII Collaboration), Chin. Phys. C 40, 113001 (2016).

[11] M. Ablikim et al. (BESIII Collaboration), Eur. Phys. J. C 76, 369 (2016).

[12] M. Ablikim et al. (BESIII Collaboration), Phys. Rev. D 92, 112008(2015).

[13] J. D. Richman and P. R. Burchat, Rev. Mod. Phys. 67, 4 (1995).

[14] P. del Amo Sanchez et al. (BABAR Collaboration), Phys. Rev. D 83, 072001 (2011).

[15] M. Ablikim et al. (BESIII Collaboration), Phys. Rev. D 94, 032001(2016).

[16] M. Ablikim et al. (BESIII Collaboration), Phys. Rev. D 92, 071101(R) (2015).

[17] M. Ablikim et al. (BESIII Collaboration), Phys. Rev. Lett. 115, 221805(2015).

[18] C. Aubin et al. (Fermilab Lattice Collaboration, MILC Collaboration, and HPQCD Collaboration), Phys. Rev. Lett. 94, 011601 (2005).

[19] L. Widhalm et al. (Belle Collaboration), Phys. Rev. Lett. 97, 061804 (2006).

[20] Y. Fang, G. Rong, H.L. Ma and J.Y. Zhao, Eur. Phys. J. C 75, 10 (2015).

[21] G. Rong, Y. Fang, H.L. Ma and J.Y. Zhao, Phys. Lett. B 743, 315(2015).

[22] C. Patrignani et al. (Particle Data Group), Chin. Phys. C 40, 100001 (2016). 